\title{
Fixed Point Theorem for Fuzzy Mappings in Generalized R - Fuzzy Metric Spaces
}

\author{
Syed Shahnawaz Ali \\ Asst. Professor \\ Sagar Institute of Research, Technology \& \\ Science, Bhopal (M.P.) \\ Anil Rajput, PhD. \\ Professor, \\ Dept. of Mathematics \\ Chandra Shekhar Azad Govt.P.G. Nodal \\ College,Sehore (M.P.)
}

\author{
Jainendra Jain, $\mathrm{PhD}$. \\ Professor, \\ Dept. of Mathematics \\ Sagar Institute of Research Technology \& \\ Science, Bhopal (M.P.) \\ D. S. Solanki, PhD. \\ Controller of Exams \\ M.P. Board of Secondary Education, Bhopal, \\ (M.P.)
}

\begin{abstract}
The study of theory of fuzzy sets was initiated by Zadeh in 1965. Since then many authors have extended and developed the theory of fuzzy sets in the fields of topology and analysis. The notion of fuzzy metric spaces has very important applications in quantum particle physics. As a result many authors have extended the Banach's Contraction Principle to fuzzy metric spaces and proved fixed point and common fixed point theorems for fuzzy metric spaces. The aim of this paper is to introduce the new definition of R-fuzzy metric space and establish a fixed point theorem for fuzzy mappings in generalized R-fuzzy metric spaces.
\end{abstract}

\section{General Terms}

Fuzzy Metric Spaces, Fixed Point Theory

\section{Keywords}

R-Fuzzy Contractive Mapping, Complete R-fuzzy Metric Space, Semi-Compatible Maps, D-Metric Space, Weak Compatibility, Common Fixed Point.

\section{INTRODUCTION}

The concept of fuzzy sets was introduced by Zadeh [13] in 1965. Since then many authors have extensively developed the theory of fuzzy sets and their applications in the fields of topology and analysis. The concept of fuzzy metric space was put forward by Kramosil and Michalek [4] in 1975. In 1994, George and Veeramani [2] extended the concept of fuzzy metric space with the help of continuous $t$-norm. Since then, many authors, viz. Gregori and Sapena [3], Mihet [5], Saadati and Park [7], Schweizer, Sherwood and Tardiff [8] etc., have proved several fixed point theorems in fuzzy metric spaces and have given various generalizations of fuzzy metric space. Dhage [1] introduced $D$ - metric space and proved some results on fixed points for a self map on complete and bounded $D$ - metric space satisfying a contraction. Rhoades [6] generalized Dhage's contractive condition by increasing the number of factors and proved the existence of a unique fixed point of a self map. The concept of $D$ - compatibility of self maps in $D$ - metric space was introduced by Singh and
Sharma [10]. They also proved some fixed point theorems using a contractive condition.

Sedgi and Shobe [9] modified the definition of $D$ - metric space and introduced $D^{*}-$ metric space and proved some basic properties in $D^{*}$ - metric spaces. Using the concept of $D^{*}$ - metric Sedgi and Shobe [9] defined $\mathcal{M}$ - fuzzy metric space and proved a common fixed point theorem in $\mathcal{M}$ fuzzy metric spaces with property (E). Another generalization of $D$ - metric space, known as generalized fuzzy metric space or $S$ - fuzzy metric space was given by Singh and Chouhan [11]. They also proved fixed point theorems for continuous mappings with a contractive condition in the generalized fuzzy metric spaces. Bijendra Singh, Shishir Jain and Shobha Jain [12] introduced the concept of semi compatibility in a fuzzy metric space.

In this paper we give a new definition of $R$ - fuzzy metric space and establish a fixed point theorem for four self maps in $R$ - fuzzy metric space satisfying an implicit relation. We recall some definitions and results in section 2 for the sake of completeness.

\section{PRELIMINARIES}

Definition 2.1: Let $X$ be a non-empty set. A generalized metric (or $D-$ metric) on $X$ is a function $D: X^{3} \rightarrow \mathbb{R}^{+}$that satisfies the following conditions for each $x, y, z, a \in X$.

(i) $\quad D(x, y, z) \geq 0$

(ii) $\quad D(x, y, z)=0$ iff $x=y=z$

(iii) $\quad D(x, y, z)=D(p\{x, y, z\})$, (symmetry), where $p$ is a permutation function.

(iv) $\quad D(x, y, z) \leq D(x, y, a)+D(a, z, z)$

The pair $(X, D)$ is called a generalized metric (or $D-$ metric) space.

Immediate examples of such a function are 
(i) $D(x, y, z)=,\max \{d(x, y), d(y, z), d(z, x)\}$,

(ii) $\quad D(x, y, z)=,d(x, y)+d(y, z)+d(z, x)$. Here $d$ is the ordinary metric on $X$.

If $X=\mathbb{R}^{n}$ then we define,

$$
D(x, y, z)=\left(\|x-y\|^{p}+\|y-z\|^{p}+\|z-x\|^{p}\right)^{1 / p},
$$

for every $\boldsymbol{\epsilon} \in \mathbb{R}^{+}$

Remark 2.1: In a $D$-metric space, we prove that $D(x, x, y)=D(x, y, y)$. For

(i) $\quad D(x, x, y) \leq D(x, x, x)+D(x, y, y)=D(x, y, y)$ and similarly

(ii) $\quad D(y, y, x) \leq D(y, y, y)+D(y, x, x)=D(y, x, x)$

Hence by (i), (ii) we get $D(x, x, y)=D(x, y, y)$.

Let $(X, D)$ be a $D-$ Metric space. For $r>0$ define

$$
B_{D}(x, r)=\{y \in X: D(x, x, y)<r\}
$$

Example 2.1: $\quad$ Let $X=\mathbb{R} . \quad D(x, y, z)=|x-y|+$ $|y-z|+|z-x|$ for all $x, y, z \in \mathbb{R}$. Thus

$$
\begin{aligned}
& B_{D}(1,2)=\{y \in \mathbb{R}: D(1, y, y)<2\} \\
& =\{y \in \mathbb{R}:|y-1|+|y-1|<2\} \\
& =\{y \in \mathbb{R}:|y-1|<1\}=(0,2) .
\end{aligned}
$$

Definition 2.2: Let $(X, D)$ be a $D-$ metric space and $A \subset X$. If for every $x \in A$ there exist $r>0$ such that $B_{D}(x, r) \subset A$, then subset $A$ is called open subset of $X$. A subset $A$ of $X$ is said to be $D-$ bounded if there exists $r>0$ such that $D(x, y, y)<r$ for all $x, y \in A$. A sequence $\left\{x_{n}\right\}$ in $X$ converges to $x$ if and only if

$D\left(x_{n}, x_{n}, x\right)=D\left(x, x, x_{n}\right) \rightarrow 0$ as $n \rightarrow \infty$. That is for each $\varepsilon>0$ there exists $n_{0} \in \mathbb{N}$ such that

For all $n \geq n_{0} \Rightarrow D\left(x, x, x_{n}\right)<\varepsilon$

This is equivalent with, for each $\varepsilon>0$ there exists $n_{0} \in \mathbb{N}$ such that

For all $n, m \geq n_{0} \Rightarrow D\left(x, x_{n}, x_{m}\right)<\varepsilon$

Indeed, if we have (1), then

$$
\begin{aligned}
D\left(x_{n}, x_{m}, x\right) & =D\left(x_{n}, x, x_{m}\right) \\
& \leq D\left(x_{n}, x, x\right)+D\left(x, x_{m}, x_{m}\right) \\
& <\frac{\varepsilon}{2}+\frac{\varepsilon}{2}=\varepsilon
\end{aligned}
$$

Conversely, set $m=n$ in (2) we have $D\left(x_{n}, x_{n}, x\right)<\varepsilon$.

Sequence $\left\{x_{n}\right\}$ in $X$ is called a Cauchy sequence if for each $\varepsilon>0$ there exist $n_{0} \in \mathbb{N}$ such that $D\left(x_{n}, x_{n}, x_{m}\right)<\varepsilon$ for each $n, m \leq n_{0}$. The $D-$ metric space $(X, D)$ is said to be complete if every Cauchy sequence is convergent

Lemma 2.1: Let $(X, D)$ be a $D$ - metric space. If $r>0$, then the ball $B_{D}(x, r)$, with center $x \in X$ and radius $r$ is an open ball.

Proof: Let $z \in B_{D}(x, r)$, hence $D(x, z, z)<r$. If we set $D(x, z, z)=\delta \quad$ and, $r^{\prime}=r-\delta$, then we prove that $B_{D}\left(z, r^{\prime}\right) \subseteq B_{D}(x, r)$. Let $y \in B_{D}(x, r)$, by triangular inequality we have

$$
\begin{aligned}
D(x, y, y)=D(y, y, x) & \leq D(y, y, z)+D(z, x, x) \\
& <r^{\prime}+\delta=r .
\end{aligned}
$$

Hence, $B_{D}\left(z, r^{\prime}\right) \subseteq B_{D}(x, r)$. That is ball $B_{D}(x, r)$ is an open ball.

Lemma 2.2: Let $(X, D)$ be a $D$-metric space. If sequence $\left\{x_{n}\right\}$ in $X$ converges to $x$, then $x$ is unique.

Proof: Let $x_{n} \rightarrow y$ and $y \neq x$. Since $\left\{x_{n}\right\}$ converges to $x$ and $y$, for each $\varepsilon>0$ there exist $n_{1} \in \mathbb{N}$ such that for every, $n \geq n_{1} \Rightarrow D\left(x, x, x_{n}\right)<\varepsilon / 2$ and $n_{2} \in \mathbb{N}$ such that for every $n \geq n_{2} \Rightarrow D\left(y, y, x_{n}\right)<\varepsilon / 2$.

If we set $n_{0}=\max \left\{n_{1}, n_{2}\right\}$ then for every $n \geq n_{0}$ by triangular inequality we have

$D(x, x, y) \leq D\left(x, x, x_{n}\right)+D\left(x_{n}, y, y\right)<\frac{\varepsilon}{2}+\frac{\varepsilon}{2}=\varepsilon$.

Hence $D(x, x, y)=0$. This is a contradiction. So, $x=y$.

Lemma 2.3: Let $(X, D)$ be a $D$-metric space. If sequence $\left\{x_{n}\right\}$ in $X$ is converges to $x$, then sequence $\left\{x_{n}\right\}$ is a Cauchy sequence.

Proof: Since $x_{n} \rightarrow x$ for each $\varepsilon>0$ there exist $n_{1} \in \mathbb{N}$ such that for every, $n \geq n_{1}$ implies that $D\left(x_{n}, x_{n}, x\right)<\varepsilon / 2$ and $n_{2} \in \mathbb{N}$ such that for every, $m \geq n_{2}$ implies that $D\left(x, x_{m}, x_{m}\right)<\varepsilon / 2$.

If we set $n_{0}=\max \left\{n_{1}, n_{2}\right\}$, then for every $n \geq n_{0}$ by triangular inequality we have

$$
\begin{aligned}
D\left(x_{n}, x_{n}, x_{m}\right) & \leq D\left(x_{n}, x_{n}, x\right)+D\left(x, x_{m}, x_{m}\right) \\
& <\frac{\varepsilon}{2}+\frac{\varepsilon}{2}=\varepsilon .
\end{aligned}
$$

Hence sequence $\left\{x_{n}\right\}$ is a Cauchy sequence. 
Definition 2.3: A function $\Delta:[0,1]^{2} \rightarrow[0,1]$ is called $t-n o r m$, if it satisfies the following conditions for any $a, b, c, d \in[0,1]$ :

$$
\begin{array}{ll}
\text { (i) } & \Delta(a, 1)=a \text { for all } a \in[0,1] ; \\
\text { (ii) } & \Delta(a, b)=\Delta(b, a) ; \\
\text { (iii) } & \Delta(a, b) \leq \Delta(c, d) \text { for } a \leq c, b \leq d \text {; } \\
\text { (iv) } & \Delta(\Delta(a, b), c)=\Delta(a, \Delta(b, c)) .
\end{array}
$$

Example 2.2: (i) $a \Delta b=a b$ for $a, b \in[0,1]$.

$$
\text { (ii) } a \Delta b=\min \{a, b\} \text {, for } a, b \in[0,1] \text {. }
$$

Definition 2.4: The triple $(X, \mathcal{M}, \Delta)$ is a fuzzy metric space if $X$ is an arbitrary set, $\Delta$ is a continuous $t-$ norm and $\mathcal{M}$ is a fuzzy set on $X^{2} \times(0, \infty)$, satisfying the following conditions:

(i) $\mathcal{M}(x, y, 0)=0$, for all $x, y \in X$;

(ii) $\quad \mathcal{M}(x, y, t)=1$, for all $t>0$ iff $x=y$ and $x, y \in$ $X$

(iii) $\quad \mathcal{M}(x, y, t)=\mathcal{M}(y, x, t) \neq 0$ for all $t>0$ and $x, y \in X$

(iv) $\quad \mathcal{M}(x, z, r+s) \geq \mathcal{M}(x, y, r) \Delta \mathcal{M}(y, z, s)$, for all $r, s>0 \& x, y, z \in X$

(v) $\quad \mathcal{M}(x, y):[0, \infty) \rightarrow[0,1]$ is left continuous;

(vi) $\quad \lim _{t \rightarrow \infty} \mathcal{M}(x, y, t)=1$, for all $t>0$ and $x, y \in$ $X$.

Example 2.3: Let $(X, d)$ be a metric space. Define $a * b=\min \{a, b\}$ and for all $x, y \in X$, and $t>0$,

$$
\mathcal{M}(x, y, t)=\frac{t}{t+d(x, y)}
$$

Then $(X, \mathcal{M}, *)$ is a fuzzy metric space. It is called the fuzzy metric space induced by the metric $d$.

Lemma 2.4: Let $\left\{y_{n}\right\}$ be a sequence in a fuzzy metric space $(X, \mathcal{M}, *)$. If there exists a number $k \in(0,1)$ such that $\mathcal{M}\left(y_{n+2}, y_{n+1}, k t\right) \geq \mathcal{M}\left(y_{n+1}, y_{n}, t\right)$, for all $t>0$, then $\left\{y_{n}\right\}$ is a Cauchy sequence in $X$.

Definition 2.5: Let $A$ and $S$ be mappings from a fuzzy metric space $(X, \mathcal{M}, *)$ into itself. Then the mappings are said to be weak compatible if they commute at their coincidence points, that is, $A x=S x$ implies that $A S x=S A x$.

Definition 2.6: Let $A$ and $S$ be mappings from a fuzzy metric space $(X, \mathcal{M}, *)$ into itself. Then the mappings are said to be compatible if

$\lim _{n \rightarrow \infty} \mathcal{M}\left(A S x_{n}, S A x_{n}, t\right)=1$, for all $t>0$,

whenever $\left\{x_{n}\right\}$ is a sequence in $X$ such that

$$
\lim _{n \rightarrow \infty} A x_{n}=\lim _{n \rightarrow \infty} S x_{n}=x \in X
$$

Definition 2.7: Let $A$ and $S$ be mappings from a fuzzy metric space $(X, \mathcal{M}, *)$ into itself. Then the mappings are said to be semi-compatible if

$\lim _{n \rightarrow \infty} \mathcal{M}\left(A S x_{n}, S x, t\right)=1$, for all $t>0$,

whenever $\left\{x_{n}\right\}$ is a sequence in $X$ such that

$\lim _{n \rightarrow \infty} A x_{n}=\lim _{n \rightarrow \infty} S x_{n}=x \in X$.

Definition 2.8: A function $\Delta:[0,1]^{3} \rightarrow[0,1]$ is called $t-n o r m$, if it satisfies the following conditions for any $a, b, c, d, e, f \in[0,1]$

(i) $\quad \Delta(a, 1,1)=a$, for all $a \in[0,1]$;

(ii) $\quad \Delta(a, b, c)=\Delta(b, c, a)=\Delta(c, a, b)$;

(iii) $\quad \Delta(a, b, c) \leq \Delta(d, e, f)$, for $a \leq d, b \leq e \& c \leq$ $f$;

(iv) $\quad \Delta(\Delta(a, b, c), d, e)=\Delta(a, \Delta(b, c, d), e)$

$$
=\Delta(a, b, \Delta(c, d, e)) \text {. }
$$

Example 2.3: (i) $a \Delta b \Delta c=a b c$ for $a, b, c \in[0,1]$.

$$
a \Delta b \Delta c=\min \{a, b, c\}
$$

for $a, b, c \in[0,1]$.

Definition 2.9: The triple $(X, S, \Delta)$ is a $S$-fuzzy metric space if $X$ is an arbitrary set, $\Delta$ is a continuous $t-n o r m$ and $S$ is a fuzzy set in $X^{3} \times(0, \infty)$ satisfying the following conditions for all $r, s, t>0$ and for all $x, y, z, w \in X$ :

$$
\begin{array}{ll}
\text { (i) } & S(x, y, z, t)>0 ; \\
\text { (ii) } & S(x, y, z, t)=1, \text { for all } t>0 \text { iff } x=y=z ; \\
\text { (iii) } & S(x, y, z, t)=S(y, z, x, t)=S(z, x, y, t) \neq 0 ; \\
\text { (iv) } & S(x, y, z, r+s+t) \\
& \quad \geq S(x, y, w, r) \Delta S(x, w, z, s) \Delta S(w, y, z, t) ; \\
\text { (v) } & S(x, y, z):[0, \infty) \rightarrow[0,1] \text { is continuous. }
\end{array}
$$

In this paper, $(X, S, \Delta)$ is considered to be the fuzzy metric space with condition.

(vi) $\quad \lim _{t \rightarrow \infty} S(x, y, z, t)=1, \quad$ for $\quad$ all $\quad x, y, z \in X$ and $t>0$.

Example 2.4: Let $(X, D)$ be a $D$ - metric space.

Define $S: X^{3} \times[0, \infty) \rightarrow[0,1]$ by

$$
S(x, y, z, t)=\frac{t}{t+d(x, y)+d(y, z)+d(z, x)}
$$

for all $t>0$ and for all $x, y, z \in X$.

Then $S$ is generalized fuzzy metric on $X$ and the triple $(X, S, \Delta)$ is generalized fuzzy metric space. 
Definition 2.10: The triple $(X, R, \Delta)$ is an $R$-fuzzy metric space if $X$ is an arbitrary set, $\Delta$ is a continuous $t-$ norm and $R$ is a fuzzy set in $X^{3} \times[0, \infty)$ satisfying the following conditions:

(i) $\quad R(x, y, z, t)>0$ for all $x, y, z \in X$;

(ii) $\quad R(x, y, z, t)=1$ for all $t>0$ iff $x=y=z$;

(iii) $\quad R(x, y, z, t)=R(y, z, x, t)=R(z, x, y, t) \neq 0$ for all $t>0$ and for all $x, y, z \in X$;

(iv) $\quad R(x, y, z, r+s+t) \geq$ $R(x, y, w, r) \Delta R(y, z, w, s) \Delta R(y, z, z, t)$ for all $r, s, t>0$ and for all $x, y, z \in X$;

(v) $\quad R(x, y, z, t):[0, \infty) \rightarrow[0,1]$ is continuous;

(vi) $\lim _{t \rightarrow \infty} R(x, y, z, t)=1$ for all $x, y, z \in X \& t>$ 0 .

Definition 2.11: The triple $(X, R, \Delta)$ is a $R$-fuzzy metric space if $X$ is an arbitrary set, $\Delta$ is a continuous $t-$ norm and $R$ is a fuzzy set in $X^{4} \times[0, \infty)$ satisfying the following conditions:

(i) $\quad R(x, y, z, w, t)>0$ for all $x, y, z, w \in X$;

(ii) $\quad R(x, y, z, w, t)=1$ for all $t>0$ iff $x=y=z=$ $w$;

(iii) $\quad R(x, y, z, w, t)=R(y, z, w, x, t)=$

$R(z, w, x, y, t)=R(w, x, y, z, t) \neq 0 \quad$ for $\quad$ all $t>0$ and for all $x, y, z, w \in X$;

(iv) $\quad R(x, y, z, w, r+s+t) \geq$

$R(x, y, z, u, r) \Delta R(y, z, w, u, s) \Delta R(y, z, w, w, t)$ for all $r, s, t>0$ and for all $x, y, z, w \in X$;

(v) $\quad R(x, y, z, w, t):[0, \infty) \rightarrow[0,1]$ is continuous;

(vi) $\lim _{t \rightarrow \infty} R(x, y, z, w, t)=1$ for all $x, y, z, w \in X$

$$
\& t>0 .
$$

Lemma 2.5: Let $(X, \mathcal{M}, \Delta)$ be a fuzzy metric space. Define $R: X^{3} \times[0, \infty) \rightarrow[0,1]$ by

$R(x, y, z)=\mathcal{M}(x, y, t) \Delta \mathcal{M}(y, z, t) \Delta \mathcal{M}(z, x, t)$, for all $x, y, z \in X$ and $t>0$. Then, $(X, R, \Delta)$ is a $R$-fuzzy metric space.

\section{Proof:}

(i) It is easy to see that $R(x, y, z, 0)=0$ for all $x, y, z \in X$, for all $t>0$;

(ii) $\quad R(x, y, z, t)=1 \quad$ iff $\quad R(x, y, t)=R(y, z, t)=$ $R(z, x, t)$ iff $x=y=z$;

(iii) $\quad R(x, y, z, t)=R(y, z, x, t)=R(z, x, y, t) \neq 0$ for all $t>0$;

(iv) $\quad R(x, y, z, r+s+t)$

$=\mathcal{M}(x, y, r+s+t) \Delta \mathcal{M}(y, z, r+s+t)$

$\Delta \mathcal{M}(z, x, r+s+t)$

$\geq \mathcal{M}(x, y, r) \Delta \mathcal{M}(y, y, s+t) \Delta \mathcal{M}(y, w, r)$

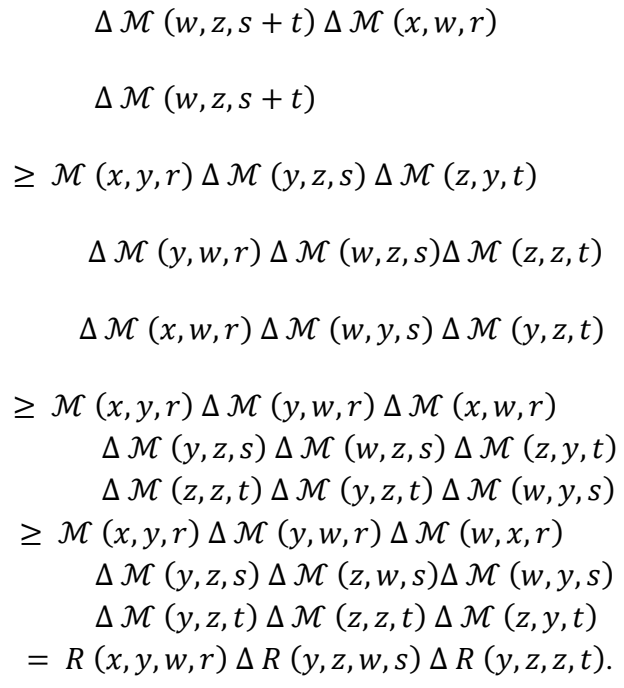

Lemma 2.6: Let $(X, \mathcal{M}, \Delta)$ be a fuzzy metric space. Define $\quad R: X^{3} \times[0, \infty) \rightarrow[0,1]$ by $R(x, y, z, w, t)=$ $\mathcal{M}(x, y, t) \Delta \mathcal{M}(y, z, t) \Delta \mathcal{M}(z, w, t) \Delta \mathcal{M}(w, x, t)$

for all $x, y, z, w \in X$ and $t>0$. Then $(X, R, \Delta)$ is an $R$ fuzzy metric space.

\section{Proof:}

(i) It is easy to see that $R(x, y, z, w, 0)=0$ for all $x, y, z, w \in X$, for all $t>0$;

(ii) $\quad R(x, y, z, w, t)=1$ iff $R(x, y, t)=R(y, z, t)=$ $R(z, w, t)=R(w, x, t)$ iff $x=y=z$;

(iii) $\quad R(x, y, z, w, t)=R(y, z, w, x, t)=$ $R(z, w, x, y, t)=R(w, x, y, z, t) \neq 0$ for all $t>0$;

(iv) $\quad R(x, y, z, w, r+s+t)$

$=\mathcal{M}(x, y, r+s+t) \Delta \mathcal{M}(y, z, r+s+t)$

$\Delta \mathcal{M}(z, w, r+s+t) \Delta \mathcal{M}(w, x, r+s+t)$

$\geq \mathcal{M}(x, y, r) \Delta \mathcal{M}(y, y, s+t) \Delta \mathcal{M}(y, z, r)$

$\Delta \mathcal{M}(z, z, s+t) \Delta \mathcal{M}(z, u, r) \Delta \mathcal{M}(u, w, s+t)$

$\Delta \mathcal{M}(x, u, r) \Delta \mathcal{M}(u, w, s+t)$

$\geq \mathcal{M}(x, y, r) \Delta \mathcal{M}(y, z, s) \Delta \mathcal{M}(z, y, t) \Delta \mathcal{M}(y, z, r)$

$\Delta \mathcal{M}(z, w, s) \Delta \mathcal{M}(w, z, t) \Delta \mathcal{M}(z, u, r) \Delta \mathcal{M}(u, w, s)$

$\Delta \mathcal{M}(w, w, t) \Delta \mathcal{M}(x, u, r) \Delta \mathcal{M}(u, y, s) \Delta \mathcal{M}(y, w, t)$

$\geq \mathcal{M}(x, y, r) \Delta \mathcal{M}(y, z, r) \Delta \mathcal{M}(z, u, r) \Delta \mathcal{M}(x, u, r)$

$\Delta \mathcal{M}(y, z, s) \Delta \mathcal{M}(z, w, s) \Delta \mathcal{M}(u, w, s) \Delta \mathcal{M}(u, y, s)$

$\Delta \mathcal{M}(z, y, t) \Delta \mathcal{M}(w, z, t) \Delta \mathcal{M}(w, w, t) \Delta \mathcal{M}(y, w, t)$

$>\mathcal{M}(x, y, r) \Delta \mathcal{M}(y, z, r) \Delta \mathcal{M}(z, u, r) \Delta \mathcal{M}(u, x, r)$

$\Delta \mathcal{M}(y, z, s) \Delta \mathcal{M}(z, w, s) \Delta \mathcal{M}(w, u, s) \Delta \mathcal{M}(u, y, s)$ 
$\Delta \mathcal{M}(y, z, t) \Delta \mathcal{M}(z, w, t) \Delta \mathcal{M}(w, w, t) \Delta \mathcal{M}(w, y, t)$

$=R(x, y, z, u, r) \Delta R(y, z, w, u, s) \Delta R(y, z, w, w, t)$.

Definition 2.12: Let $A$ and $S$ be mappings from a fuzzy metric space $(X, \mathcal{M}, \Delta)$ into itself. We say that $A$ and $B$ satisfy the property (E), if there exists a sequence $\left\{x_{n}\right\}$ such that

$$
\lim _{n \rightarrow \infty} R\left(A x_{n}, u, u, t\right)=\lim _{n \rightarrow \infty} R\left(B x_{n}, u, u, t\right)=1
$$

for some $u \in X$ and $t>0$.

\section{MAIN THEOREM}

\subsection{A Class of Implicit Relations}

Let $\Phi$ denotes a family of mappings such that $\operatorname{each} \varphi$ : $[0,1]^{20} \rightarrow[0,1]$, and $\varphi$ is continuous and increasing in each co-ordinate variable. Also $\varphi(s, s, \ldots, s)>s$ for every $s \in[0,1)$.

Example 3.1: Let $\varphi:[0,1]^{20} \rightarrow[0,1]$ be defined by $\varphi\left(x_{1}, x_{2}, \ldots, x_{20}\right)=\left(\min \left\{x_{i}\right\}\right)_{h}$, for some $0<h<1$.

Theorem 3.1: Let $A, B, S$ and $T$ be self - mappings of an $R$ - fuzzy metric space $(X, R, *)$ satisfying:

(i) $A(X) \subseteq T(X), B(X) \subseteq S(X)$ and $T(X)$ or $S(X)$ is a complete fuzzy metric subspace of $X$.

(ii) The pair $(A, S)$ and $(B, T)$ are weakly compatible and $(A, S)$ or $(B, T)$ satisfy the property $(E)$.

(iii) $R(A x, B y, B z, t)$

$\geq \varphi\{R(S x, T y, T z, k t), R(S x, B y, T z, k t), R(S x, T y, B z, k t)$, $R(S x, B y, B z, k t), R(S x, B y, B y, k t), R(S x, T y, T y, k t)$, $R(S x, B z, B z, k t), R(S x, T z, T z, k t), R(T y, B y, B z, k t)$, $R(T y, T y, B z, k t), R(T y, B y, B y, k t), R(T y, B z, B z, k t)$, $R(B y, T y, T z, k t), R(B y, B y, T z, k t), R(B y, T z, T z, k t)$, $R(T z, B z, B z, k t), R(T z, B z, B y, k t), R(B y, T y, T y, k t)$, $R(B z, T z, T y, k t), R(B z, T z, T z, k t)\}$.

Then $A, B, S$ and $T$ have a unique common fixed point in $X$.

Proof: Let the pair $(B, T)$ satisfying property $(E)$, hence there exist a sequence $\left\{x_{n}\right\}$ such that,

$$
\lim _{n \rightarrow \infty} R\left(B x_{n}, u, u, t\right)=\lim _{n \rightarrow \infty} R\left(T x_{n}, u, u, t\right)=1
$$

for some $u \in X$ and every $t>0$. As $\subseteq S X$, there exists a sequence $\left\{y_{n}\right\}$ such that $B x_{n}=S y_{n}$, hence $\lim _{n \rightarrow \infty} R\left(S y_{n}, u, u, t\right)=1$.

We prove that $\lim _{n \rightarrow \infty} R\left(A y_{n}, u, u, t\right)=1$. Since,

$R\left(A y_{n}, B x_{n}, B x_{n+1}, t\right)$

$\geq \varphi\left\{R\left(S y_{n}, T x_{n}, T x_{n+1}, k t\right), R\left(S y_{n}, B x_{n}, T x_{n+1}, k t\right)\right.$,

$R\left(S y_{n}, T x_{n}, B x_{n+1}, k t\right), R\left(S y_{n}, B x_{n}, B x_{n+1}, k t\right)$,

$R\left(S y_{n}, B x_{n}, B x_{n}, k t\right), R\left(S y_{n}, T x_{n}, T x_{n}, k t\right)$

$R\left(S y_{n}, B x_{n+1}, B x_{n+1}, k t\right), R\left(S y_{n}, T x_{n+1}, T x_{n+1}, k t\right)$

$R\left(T x_{n}, B x_{n}, B x_{n+1}, k t\right), R\left(T x_{n}, T x_{n}, B x_{n+1}, k t\right)$

$R\left(T x_{n}, B x_{n}, B x_{n}, k t\right), R\left(T x_{n}, B x_{n+1}, B x_{n+1}, k t\right)$

$R\left(B x_{n}, T x_{n}, T x_{n+1}, k t\right), R\left(B x_{n}, B x_{n}, T x_{n+1}, k t\right)$

$R\left(B x_{n}, T x_{n+1}, T x_{n+1}, k t\right), R\left(T x_{n+1}, B x_{n+1}, B x_{n+1}, k t\right)$,

$R\left(T x_{n+1}, B x_{n+1}, B x_{n}, k t\right), R\left(B x_{n}, T x_{n}, T x_{n}, k t\right)$

$\left.R\left(B x_{n+1}, T x_{n+1}, T x_{n}, k t,\right), R\left(B x_{n+1}, T x_{n+1}, T x_{n+1}, k t\right)\right\}$

Taking $n \rightarrow \infty$ in the above inequality, we get

$\lim _{n \rightarrow \infty} R\left(A y_{n}, B x_{n}, B x_{n+1}, t\right)$

$\geq \varphi(R(u, u, u, k t), R(u, u, u, k t), \cdots, R(u, u, u, k t))$

$=1$

Therefore, $\lim _{n \rightarrow \infty} R\left(A y_{n}, u, u, t\right)=1$. Hence

$\lim _{n \rightarrow \infty} A y_{n}=\lim _{n \rightarrow \infty} S y_{n}=\lim _{n \rightarrow \infty} B x_{n}=$ $\lim _{n \rightarrow \infty} T x_{n}=u$.

Let $S(X)$ be complete $R$ - fuzzy metric space, then there exists $x \in X$ such that $S x=u$. If $A x \neq u$, then we have

$R\left(A x, B x_{n}, B x_{n+1}, t\right)$

$\geq \varphi\left\{R\left(S x, T x_{n}, T x_{n+1}, k t\right), R\left(S x, B x_{n}, T x_{n+1}, k t\right)\right.$,

$R\left(S x, T x_{n}, B x_{n+1}, k t\right), R\left(S x, B x_{n}, B x_{n+1}, k t\right)$,

$R\left(S x, B x_{n}, B x_{n}, k t\right), R\left(S x, T x_{n}, T x_{n}, k t\right)$

$R\left(S x, B x_{n+1}, B x_{n+1}, k t\right), R\left(S x, T x_{n+1}, T x_{n+1}, k t\right)$

$R\left(T x_{n}, B x_{n}, B x_{n+1}, k t\right), R\left(T x_{n}, T x_{n}, B x_{n+1}, k t\right)$,

$R\left(T x_{n}, B x_{n}, B x_{n}, k t\right), R\left(T x_{n}, B x_{n+1}, B x_{n+1}, k t\right)$,

$R\left(B x_{n}, T x_{n}, T x_{n+1}, k t\right), R\left(B x_{n}, B x_{n}, T x_{n+1}, k t\right)$,

$R\left(B x_{n}, T x_{n+1}, T x_{n+1}, k t\right), \quad R\left(T x_{n+1}, B x_{n+1}, B x_{n+1}, k t\right)$, 


$$
\begin{aligned}
& R\left(T x_{n+1}, B x_{n+1}, B x_{n}, k t\right), R\left(B x_{n}, T x_{n}, T x_{n}, k t\right), \\
& \left.R\left(B x_{n+1}, T x_{n+1}, T x_{n}, k,\right), R\left(B x_{n+1}, T x_{n+1}, T x_{n+1}, k t\right)\right\} .
\end{aligned}
$$

Taking $n \rightarrow \infty$ on the above inequality, we get $R(A x, u, u, t)=1$, hence

$A x=u=S x$. By $(A, S)$ be weakly compatible, we have $A S x=S A x$, so

$$
A A x=A S x=S A x=S S x .
$$

As $A X \subset T X$, there exist $v \in X$ such that $A x=T v$. We prove that

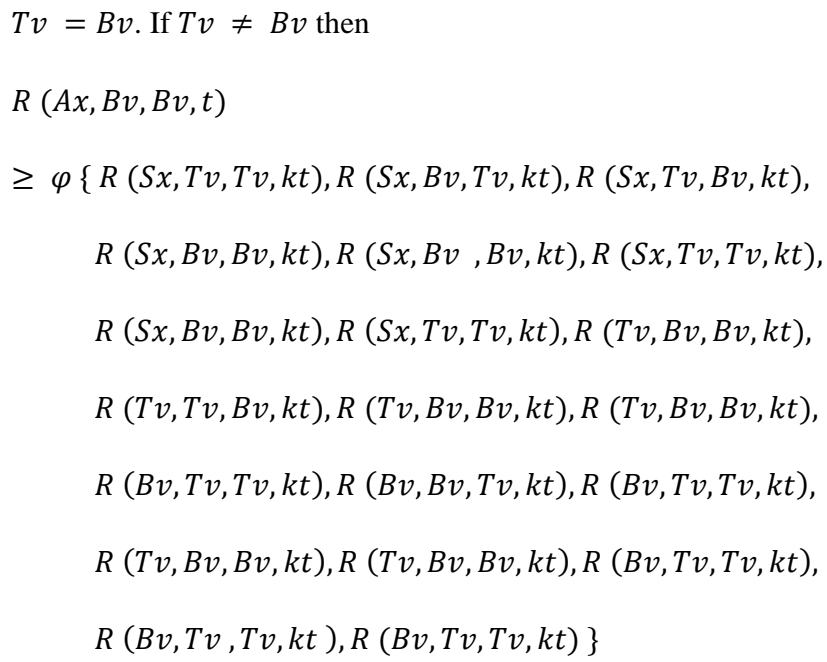

If $A u \neq u$, then

$$
R(A u, u, u, t)>R(A u, u, u, k t)
$$

is a contradiction. Thus, $A y=S u=u$. Now, we prove $B u=u$.
$R(u, B u, B u, t)=R(A u, B u, B u, t)$

$\geq \varphi\{R(S u, T u, T u, k t), R(S u, B u, T u, k t), R(S u, T u, B u, k t)$, $R(S u, B u, B u, k t), R(S u, B u, B u, k t), R(S u, T u, T u, k t)$, $R(S u, B u, B u, k t), R(S u, T u, T u, k t), R(T u, B u, B u, k t)$, $R(T u, T u, B u, k t), R(T u, B u, B u, k t), R(T u, B u, B u, k t)$, $R(B u, T u, T u, k t), R(B u, B u, T u, k t), R(B u, T u, T u, k t)$, $R(T u, B u, B u, k t), R(T u, B u, B u, k t), R(B u, T u, T u, k t)$, $R(B u, T u, T u, k t), \quad R(B u, T v, T u, k t)\}$

.If $B u \neq u$, then

$R(u, B u, B u, t)>R(u, B u, B u, k t)$

is a contradiction. Thus $=B u=S u=T u=u$. So $A, B, S$ and $T$ have a common fixed point $u$.

Now to prove uniqueness, if possible $v \neq u$ is another common fixed point of $A, B, S$ and $T$. Then

$R(v, u, u, t)=R(A v, B u, B u, t)$

$\geq \varphi\{R(S v, T u, T u, k t), R(S v, B u, T u, k t), R(S v, T u, B u, k t)$,

$R(S v, B u, B u, k t), R(S v, B u, B u, k t), R(S v, T u, T u, k t)$, $R(S v, B u, B u, k t), R(S v, T u, T u, k t), R(T u, B u, B u, k t)$, $R(T u, T u, B u, k t), R(T u, B u, B u, k t), R(T u, B u, B u, k t)$, $R(B u, T u, T u, k t), R(B u, B u, T u, k t), R(B u, T u, T u, k t)$, $R(T u, B u, B u, k t), R(T u, B u, B u, k t), R(B u, T u, T u, k t)$, $R(B u, T u, T u, k t), R(B u, T v, T u, k t)\}$

This implies that $R(v, u, u, t)>R(v, u, u, k t)$.

This is contradiction. Hence $u=v$.

So $A, B, S$ and $T$ have a unique common fixed point $u$

\section{CONCLUSION}

Fuzzy set theory and Fuzzy Fixed Point Theory has numerous applications in applied sciences and engineering such as neural network theory, stability theory, mathematical programming, modeling theory, medical sciences (medical genetics, nervous system), image processing, control theory, communications etc. As a result fuzzy fixed point theory has become an area of interest for specialists in fixed point theory. In this paper we have given a new definition of $\mathrm{R}-$ Fuzzy metric space and proved a common fixed point theorem for four self mappings in $\mathrm{R}$ - Fuzzy metric spaces satisfying a class of implicit relations. The result can be extended for more 
number of self mappings satisfying a more complex class of implicit relations.

\section{REFERENCES}

[1]. Dhage B.C. 1992. "Generalized Metric Spaces and Mappings with Fixed Point". Bull. Calcutta Math. Soc., vol. 84(4), pp. 329-336.

[2]. George A. and Veeramani P. 1994. "On Some Results in Fuzzy Metric Spaces”. Fuzzy Sets and Systems, vol. 64, pp. 395-399.

[3]. Gregori V. and Sapena A. 2002. "On Fixed Point Theorem in Fuzzy Metric Spaces". Fuzzy Sets and Systems, vol. 125, pp. 245-252.

[4]. Kramosil I. and Michalek J. 1975. "Fuzzy Metric and Statistical Metric Spaces”. Kybernetica, vol. 11, pp. 326334.

[5]. Mihet D. 2004. "A Banach Contraction Theorem in Fuzzy Metric Spaces”. Fuzzy Sets and Systems, vol. 144, pp. 431-439.

[6]. Rhoades B.E. 1996. "A Fixed Point Theorem for Generalized Metric Spaces”. Int. J. Math. Math. Sci., vol. 19(1), pp. 145-153.
[7]. Saadati R. and Park J. H. 2006. "On the Intuitionistic Fuzzy Topological Spaces". Chaos, Solitons and Fractals, vol. 27, pp. 331-344.

[8]. Schweizer B., Sherwood H., and Tardiff R.M. 1988 "Contractions on PM-Space Examples and Counterexamples”. Stochastica, vol.1, pp. 5-17.

[9]. Sedghi S. and Shobe N. 2006. "Fixed Point Theorem in M-Fuzzy Metric Spaces with Property (E)". Advances in Fuzzy Mathematics, vol. 1, pp. 55-65.

[10].Singh B. and Sharma R.K. 2002. "Common Fixed Points via Compatible Maps in D-Metric Spaces". Rad. Mat., vol. 11(1), pp. 145-153.

[11].Singh Bijendra and Chouhan M. S. 1997. "Generalized Fuzzy Metric Space and Fixed Point Theorem". Bull. Calcutta Math. Soc., vol. 89, pp. 457-460.

[12]. Singh Bijendra, Jain Shishir and Jain Shobha. 2006. "Semi Compatibility and Common Fixed Point in Fuzzy Metric Space". Bull. Calcutta Math. Soc., vol. 98, pp 229-236.

[13].Zadeh L. 1965. "Fuzzy Sets". Inform and control, vol. 8, pp. 338-353. 